Revista de la Escuela de Ciencias de la Educación, año 14, nRo. 13, vol. 2, julio a diciembre de 2018. Páginas 53-68. ISSN 1851-6297 (DESDE DICIEMBRE DE 2006 A DICIEMBRE DE 2017). ISSN 2362-3349 (EN LINNEA). ANÁLISIS DEL SISTEMA DE

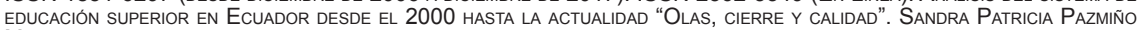
Moscoso.

\title{
ANÁLISIS DEL SISTEMA DE EDUCACIÓN SUPERIOR EN ECUADOR DESDE EL 2000 HASTA LA ACTUALIDAD "OLAS, CIERRE Y CALIDAD"
}

\author{
Sandra Patricia Pazmiño Moscoso* \\ Universidad Central de Ecuador, Ecuador \\ sppazmino@uce.edu.ec
}

Recibido: 1/10/2017 Aceptado: 10/01/2018

\section{Resumen}

Se explora la constelación de estímulos e intereses que hicieron posible la creación, sin la planificación ni los controles de calidad adecuados, de una cantidad sin precedentes de universidades durante los años 1990 e inicios de 2000. Luego se examina el proceso político y legal que permitió la depuración de instituciones de educación superior de mala calidad: desde el Mandato Constituyente $\mathrm{N}^{\circ} 14$ en 2008 hasta la resolución final del Consejo de Evaluación Acreditación y Aseguramiento de la Calidad de la Educación Superior (CEAACES) en 2012.

Ecuador como consecuencia de la Constitución aprobada en octubre del 2008 ha iniciado un nuevo proceso de institucionalización, donde la acción más relevante es la expedición de la Ley de Educación Superior dos años después en el año 2010.

Los fines del sistema nacional de educación no son más que el desarrollo de las potencialidades individuales y colectivas, en un entorno de aprendizaje y generación de conocimiento y cultura (Bourdieu, 1993).

Actualmente la sociedad atraviesa cambios constantes dentro de los que la educación no queda de lado, siendo necesaria la reforma de conocimientos

* Es Licenciada en Radiología, actualmente, docente de la Facultad de Ciencias Médicas de la Universidad Central del Ecuador, en las asignaturas de Primeros Auxilios, Supervisión de Prácticas Hospitalarias en la Carrera de Radiología, además de aportar en los procesos de Acreditación de las Carreras que conforman la Facultad de Ciencias Médicas y Asistencia en la Gestión Universitaria. Se encuentra cursando la Maestría en Educación con acentuación en Tecnología Educativa en el Instituto Tecnológico y de Estudios Superiores de Monterrey y el Doctorado en Humanidades y Artes, mención Ciencias de la Educación en la Universidad Nacional de Rosario. 
Revista de la Escuela de Ciencias de la Educación, año 14, nRo. 13, vol. 2, julio a diciembre de 2018. PÁginas 53-68. ISSN 1851-6297 (DESDE DICIEMBRE DE 2006 A DICIEMBRE DE 2017). ISSN 2362-3349 (EN LINEA). ANÁLISIS DEL SISTEMA DE

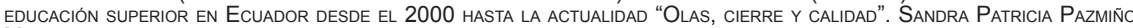
Moscoso.

que se adapten a la practicidad desde el salón de clase como punto de inicio.

Los procesos de aseguramiento de la calidad van más lejos de representar simplemente un mecanismo institucional, en ellos se expresa el poder de transformación que tiene el Derecho cuando se sintoniza con el beneficio colectivo mas no con intereses que involucren conflictos de poder y fines de lucro.

Palabras clave: Sistema de Educación Superior - Calidad - Ciencia - Educación - Profesional.

\section{Abstract}

It explores the constellation of stimuli and interests that made possible the creation, without proper planning or quality controls, of an unprecedented amount of universities during the 1990s and early 2000s. Then, the political and legal process that allowed the purging of high quality institutions of higher education: from the Constituent Mandate No. 14 in 2008 to the final resolution of the Evaluation Council Accreditation and Quality Assurance of Higher Education (CEAACES) in 2012.

Ecuador as a consequence of the Constitution approved in October 2008 has initiated a new process of institutionalization, where the most relevant action is the issuance of the Higher Education Law two years later in 2010. The purposes of the national education system are no more than the development of individual and collective potential, in an environment of learning and generation of knowledge and culture (Bourdieu, 1993).

Currently, society is going through constant changes within which education is not left aside, being necessary the reform of knowledge that adapts to practicality from the classroom as a starting point. Quality assurance processes go beyond simply representing an institutional mechanism, they express the power of transformation that the law has when it is tuned to the collective benefit but not with interests that involve conflicts of power and profit.

Keywords: Higher Education System - Quality - Science - Education Professional.

\section{Introducción}

En la actualidad la Educación Superior en Ecuador afronta diversos retos como la reestructuración económica, renovación del Gobierno y políticas que desencadenan en procesos de modernización en el contexto institucional.

Ecuador es un país que los últimos años mediante diferentes mecanismos jurídicos como la creación de leyes y nuevas organizaciones gubernamentales desean mejorar el sistema de educación superior.

Por esta razón se generan nuevos retos relacionados con la gestión y 
Revista de la Escuela de Ciencias de la Educación, año 14, nRo. 13, vol. 2, julio a diciembre de 2018. Páginas 53-68. ISSN 1851-6297 (DESDE DICIEMBRE DE 2006 A DICIEMBRE DE 2017). ISSN 2362-3349 (EN LINNEA). ANÁLISIS DEL SISTEMA DE

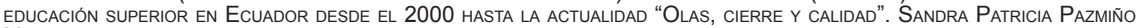
Moscoso.

administración de Instituciones Educativas enfocados a problemáticas como presencia de movimientos étnicos, raciales, diversidad de género, prevalencia de la pobreza, deuda externa, deterioro de recursos naturales y reducción de presupuesto a la Educación Superior.

\section{Materiales y Métodos}

El estudio se realizó identificando los principales agentes que influyen en el desarrollo de la Educación Superior, tomando en cuenta las Instituciones Públicas, para estatales y privadas con y sin fines de lucro del Ecuador, relacionadas con el tema que nos ocupa.

Se obtuvo información de fuentes primarias y secundarias. En el primer caso se trabajaron bases de datos de las instituciones públicas, semi estatales y privadas con y sin fines de lucro, y se trabajó un enfoque funcional, con el marco analítico de las Políticas Universitarias.

Los ámbitos de información trabajados se relacionaron con los siguientes aspectos: apertura de Instituciones de Educación Superior, evaluación de Calidad, repercusión de las Políticas de la época.

\section{Resultados y discusión \\ Los antecedentes}

Mencionando los organismos del Sistema de Educación Superior, a través de la Constitución Política de 1998 se dispuso la creación de un Sistema Autónomo de Evaluación y Acreditación para asegurar los objetivos de calidad de las instituciones.

De acuerdo al Art 13 de la Ley de Educación Superior era atribución del CONESUP (Consejo de Educación Superior) definir las políticas de formación profesional, investigación científica y tecnológica, de vinculación con la colectividad y de colaboración nacional e internacional ${ }^{1}$; la Secretaría Técnica siendo el órgano regulador de las políticas de planificación, regulación y control que emite el CONESUP en el ámbito del Sistema Nacional de Educación Superior.

Posteriormente, en el año 2000, a través de la Ley Orgánica de Educación Superior-LOES se creó el Consejo Nacional de Evaluación y Acreditación-CONEA para dirigir dicho sistema y trabajar en la autoevaluación institucional, la evaluación externa y la acreditación. EI CONEA empieza su funcionamiento en el año 2002.

Tiempo después a través del Decreto Ejecutivo 517, el Presidente de la República Rafael Correa, fusionó a la Secretaría Nacional de Ciencia y Tecnología (Senacyt) con la Secretaría Nacional de Educación Superior, Ciencia y Tecnología e Innovación, creada en la nueva Ley de Educación Superior en vigencia desde el 12 de octubre del 2010.

Con la vigencia del nuevo cuerpo legal; las funciones, el presupuesto, equipamiento y demás bienes activos del CONESUP, pasaron al nuevo orga- 
Revista de la Escuela de Ciencias de la Educación, año 14, nRo. 13, vol. 2, julio a diciembre de 2018. PÁginas 53-68. ISSN 1851-6297 (DESDE DICIEMBRE DE 2006 A DICIEMBRE DE 2017). ISSN 2362-3349 (EN LINEA). ANÁLISIS DEL SISTEMA DE

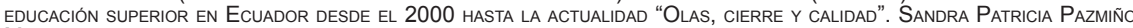
Moscoso.

nismo que lo reemplazó.

Se estableció la gratuidad de la educación superior pública, eliminó al CONESUP y dividió las funciones que este organismo tenía, creando tres organismos diferentes: CES (Consejo de Educación Superior), SENESCYT (Secretaría Nacional de Educación Superior, Ciencia, Tecnología e Innovación) y CEAACES (Consejo de Evaluación, Acreditación y Aseguramiento de la Calidad de la Educación Superior).

El Mandato Constituyente No. 14, expedido por la Asamblea Nacional Constituyente el 22 de julio de 2008, establece la obligación del Consejo Nacional de Evaluación y Acreditación (CONEA) de elaborar un informe técnico sobre el nivel de desempeño institucional de los establecimientos de educación superior (1), a fin de garantizar su calidad, propiciando su depuración y mejoramiento. Dicha evaluación obtuvo los siguientes resultados:

En primer lugar, se exploró la constelación de estímulos e intereses que hicieron posible la creación, sin la planificación ni los controles de calidad adecuados, de una cantidad sin precedentes de universidades durante los años 1990 e inicios de 2000. Luego se examinó el proceso político y legal que permitió la depuración de instituciones de educación superior de mala calidad: desde el Mandato Constituyente $\mathrm{N}^{\circ} 14$ en 2008 hasta la resolución final del Consejo de Evaluación Acreditación y Aseguramiento de la Calidad de la Educación Superior (CEAACES) en 2012 (2).

Se crearon sin control varias universidades sin planificación ni políticas de control de calidad, el 12 de abril del 2012 Ecuador contaba con 71 universidades, 45 de ellas creadas entre 1992 y 2006, lo que evidencia un crecimiento del $273 \%$ en apenas 14 años.

Pero el fenómeno más generalizado durante el periodo neoliberal fue, sin duda, la creación de un gran número de instituciones privadas, especialmente de carácter autofinanciado, cuyos ingresos económicos se basaban en el arancelamiento de sus actividades académicas y programas formativos. De las 45 universidades creadas entre 1992 y 2006, 35 fueron instituciones de este tipo.

Existió una segunda ola de creación de Instituciones de Educación Superior, esta vez con la característica de ser privadas y de élite y producto de la mesocratización de las entidades de Educación Publicas, sectores de las clases sociales altas buscaron espacios menos "politizados", conflictivos y de mejor prestigio, con la finalidad de prolongar la reproducción cultural e ideología de su descendencia.

Contrariamente a la primera ola, esta fue menos orientada a la modernidad conservadora hispana latinoamericana y mayormente influenciada por la estérica del modelo universitario estadounidense. 
Revista de la Escuela de Ciencias de la Educación, año 14, nRo. 13, vol. 2, julio a diciembre de 2018. Páginas 53-68. ISSN 1851-6297 (DESDE DICIEMBRE DE 2006 A DICIEMBRE DE 2017). ISSN 2362-3349 (EN LÍNEA). ANÁLISIS DEL SISTEMA DE

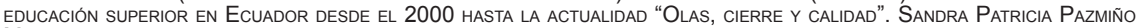
Moscoso.

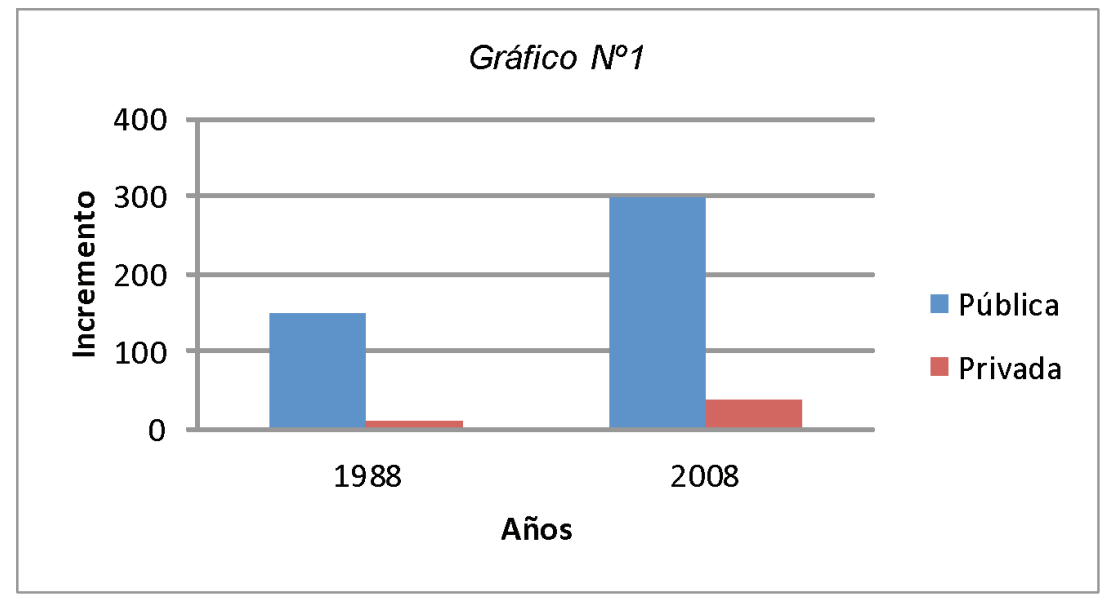

Gráfico №1: Incremento de matrícula de estudiantes

en universidades privadas (1998 - 2008).

Fuente: INEC

Elaboración: CEAACES

Finalmente existió una tercera ola de creación de universidades privadas, misma que fue masiva como resultado de la no regulación neoliberal, alternativa no elitista que su finalidad era suplir la creciente demanda en el contexto de una Institución de Educación Superior pública con grandes dificultades para absorberla.

Estas instituciones hasta cierto punto sin barreras académicas de admisión, con un costo menos elevado de otras más elitistas con significativas carencias de calidad fueron las que se convertirían en las Universidades de categoría "E".

La característica más preponderante de la tercera ola de creación de universidades privadas fue el papel del mercado y afán de lucro, muchas de estas no fueron creadas en busca de suplir una oferta que no existía sino al contrario, por competir en lugares donde ya existía como antecedente un excedente de oferta académica.

La ciudad de Quito, capital del Ecuador fue uno de los focos con mayor crecimiento de oferta universitaria, en ésta y sus alrededores se crearon 18 de las 45 universidades creadas entre 1992 y 2006, y 7 de las 14 cerradas en abril del 2012.

Con estrategias de marketing, las instituciones de la tercera ola empezaron a ofertar carreras con nombres más atractivos sin fondo académico, una forma de atraer al estudiante "cliente", en un ambiente donde "ingeniero" llego 
Revista de la Escuela de Ciencias de la Educación, año 14, nRo. 13, vol. 2, julio a diciembre de 2018. PÁginas 53-68. ISSN 1851-6297 (DESDE DICIEMBRE DE 2006 A DICIEMBRE DE 2017). ISSN 2362-3349 (EN LINEA). ANÁLISIS DEL SISTEMA DE

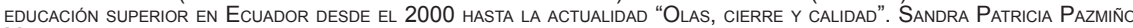
Moscoso.

a ser la palabra mayormente dotada de prestigio que "licenciado", incluso en ámbitos alejados a los que cubre las ingenierías; por ejemplo: Ingeniería en Negocios Internacionales o Ingeniería en Talento Humano.

A esto se debe sumar la crisis económica de 1999 (Long, 2013, p. 13) que fue el resultado de un conjunto de problemas acumulados, se manifestó de manera especial a través de las quiebras bancarias facilitadas con la creación en 1998 de la Agencia de Garantía de Depósitos (AGD) y a través de la dolarización, problemas que tuvieron su desenlace en el derrocamiento del presidente Jamil Mahuad en enero de 2000.

\section{Tabla 1}

Sistema de Educación Superior del Ecuador 2008

\begin{tabular}{|c|c|c|c|c|c|}
\hline Creación & Universidades & $\begin{array}{c}\text { Escuelas } \\
\text { Politécnicas }\end{array}$ & Públicas & Cofinanciadas & Autofinanciadas \\
\hline $1826-1989$ & 21 & 4 & 19 & 6 & \\
\hline $1990-2000$ & 28 & 4 & 6 & 3 & 23 \\
\hline $2001-2006$ & 15 & & 4 & & 11 \\
\hline TOTAL & 64 & 5 & 29 & 9 & 34 \\
\hline
\end{tabular}

Tabla 1: Muestra las cifras correspondientes a la creación de Universidades,

Escuelas Politécnicas, IES Públicas, Cofinanciadas y Autofinanciadas, relación 1826-2006.

El sistema contiene también 369 INSTITUTOS SUPERIORES registrados, de los cuales estaban a esa fecha en funcionamiento 287. En el análisis de la presente investigación no se consideraran a los Institutos Superiores. Fuente: CONEA

\section{La categorización de universidades}

En 2008 la Asamblea Constituyente de Montecristi construyó la base para dar un cambio en la conformación del Sistema de Educación Superior, recuperándola como un bien público, donde rigen los principios de: autonomía responsable refiriéndola a la garantía absoluta en el ejercicio de la academia sin obviar calidad y rendición de cuentas, cogobierno, integralidad, calidad y pertinencia social, implicando que el Sistema de Educación Superior actúe conforme los nuevos desafíos del país, necesidades del entorno social y objetivos; todo esto cumpliendo leyes y reglamentos.

Por lo que se expidió, además, el Mandato Constituyente $N^{\circ} 14$. Los artículos primero y segundo de dicho mandato decretaron la derogación es decir, la extinción de la ley de creación de la Universidad Cooperativa 
Revista de la Escuela de Ciencias de la Educación, año 14, nRo. 13, vol. 2, julio a diciembre de 2018. Páginas 53-68. ISSN 1851-6297 (DESDE DICIEMBRE DE 2006 A DICIEMBRE DE 2017). ISSN 2362-3349 (EN LINNEA). ANÁLISIS DEL SISTEMA DE

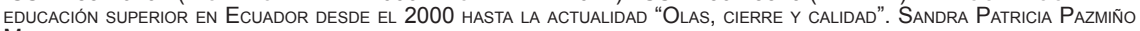
Moscoso.

de Colombia por haber detectado "irregularidades permanentes"2, fundamentalmente la venta -apenas disfrazada- de títulos. Este hecho abriría una verdadera caja de Pandora sobre la existencia de un gran número de "universidades" con prácticas similares y pobre calidad académica.

Lo que resultó retrospectivamente aún más trascendente que el cierre de la Universidad Cooperativa de Colombia fue que el Mandato 14 le encargó a los entes reguladores de la educación superior realizar un diagnóstico sobre el estado del Sistema: al Consejo Nacional de Educación Superior (CONESUP) le encomendó determinar "la situación académica y jurídica de todas las entidades educativas bajo su control"', y al Consejo Nacional de Evaluación y Acreditación (CONEA) le solicitó "un informe técnico sobre el nivel de desempeño institucional de los establecimientos de educación superior, a fin de garantizar su calidad, propiciando su depuración y mejoramiento.

Del informe del CONESUP, hoy poco se habla. Fue entregado a destiempo y con vacíos quedando absolutamente deslegitimado en el transcurso de los años. Por otro lado, el informe final del CONEA, entregado el 4 de noviembre del 2009, marcaría durante los años posteriores al Sistema de Educación Superior ecuatoriano. Era la primera vez que una institución ecuatoriana realizaba una intervención evaluativa a todo el sistema para emitir un diagnóstico sobre las grandes tendencias sistémicas, así como una apreciación de la situación individual de las instituciones, tanto universidades y escuelas politécnicas (en un informe de 194 páginas), cuanto institutos superiores técnicos y tecnológicos (en un informe de 123 páginas). Entre otras cosas, el informe del CONEA dictaminó lo siguiente: "...La universidad ecuatoriana se evidencia, a lo largo de este informe, como un conjunto fragmentado por múltiples brechas: académica, democrática, investigativa, tecnológica, brechas que, a su vez, expresan múltiples fenómenos" (3).

Adicionalmente, y polémicamente, el informe del CONEA clasificó a todas las universidades y escuelas politécnicas en cinco categorías (A, B, C, D y E) de acuerdo a su nivel de desempeño respecto de determinados parámetros de evaluación. Identificó a un conjunto de 26 universidades cuyo desempeño reveló ser claramente deficiente, y las ubicó en la categoría "E", describiéndolas de la siguiente manera.

Estas universidades (Categoría "E") representan al sector más dinámico y de crecimiento más rápido en la educación universitaria del Ecuador en los últimos años. Todas estas universidades han sido creadas en los últimos 12 años, y, 14 de ellas, a partir de la entrada en vigencia de la Ley Orgánica de Educación Superior (LOES; mayo 2000). Se trata de instituciones que, definitivamente, no presentan las condiciones que exige el funcionamiento de una institución universitaria y en las que se evidencian 
Revista de la Escuela de Ciencias de la Educación, año 14, nRo. 13, vol. 2, julio a diciembre de 2018. PÁginas 53-68. ISSN 1851-6297 (DESDE DICIEMBRE DE 2006 A DICIEMBRE DE 2017). ISSN 2362-3349 (EN LINEA). ANÁLISIS DEL SISTEMA DE

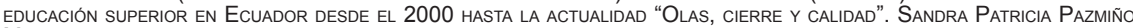
Moscoso.

las deficiencias y problemas que afectan a la universidad ecuatoriana... la actividad y permanencia de la planta docente se sustentan en prácticas altamente precarizadas del ejercicio de la docencia. La oferta académica de este grupo de universidades está concentrada en carreras como administración, contabilidad y auditoría, gestión empresarial, mercadotecnia, gestión turística, relaciones públicas y otras con denominaciones "curiosas e ingeniosas" que, como se señaló anteriormente, normalmente no corresponden a una oferta académica universitaria...Se trata de locales (oficinas, departamentos, casas de vivienda) precariamente adaptados para acoger a una población estudiantil y que carecen de las facilidades indispensables y de la funcionalidad elemental que exige el desarrollo de las actividades académicas. [...] En cumplimiento de lo establecido en el Mandato 14, este informe recomienda a la Asamblea Legislativa, depurar el sistema universitario ecuatoriano de este grupo de universidades, a través de las acciones legales que están en el marco de competencia de la Asamblea, precautelando los derechos de las y los estudiantes (4).

Inexplicablemente la finalidad de depuración de las 26 universidades categoría "E" quedó en nada; se convirtió en una especie de "piedrita" para una Asamblea Nacional inmersa en una agenda legislativa llena de problemas que puso como primera prioridad aterrizar los principios constitucionales en leyes y códigos orgánicos.

Sin embargo, en el año 2010 retomó el tema de la educación superior a debate, debido a la necesidad de expedir una nueva LOES - Ley Orgánica de Educación Superior que concrete los principios de la Constitución de 2008 en el nuevo marco normativo ecuatoriano.

Esta LOES, fue aprobada después de varios debates en el gobierno, en especial a la Secretaría Nacional de Planificación y Desarrollo (SENPLADES) con su corporación a la Educación Superior.

Varios rectores presentaron una rotunda oposición a la nueva propuesta de ley, ellos en defensa de una visión de autonomía universitaria, profundamente anclada en el ultra-autonomismo neoliberal, rechazando "lo público" y a su vez aunque sin conciencia académica de aquello los pilares más elementales del contrato social.

Fue una de las leyes post-Montecristi más disputadas dentro y fuera del espacio legislativo. En pocos otros ámbitos de debate político, se pudo vislumbrar tan alto nivel de corporativismo y espíritu de cuerpo del gremio de los rectores.

La Asamblea Nacional expidió, el 12 de octubre de 2010, la nueva Ley Orgánica de Educación Superior (LOES) misma que reafirmó la gratuidad de la educación superior; ; reiteró la necesidad de articular la educación con las metas de desarrollo local y nacional; planteó la necesidad de ase- 
Revista de la Escuela de Ciencias de la Educación, año 14, nRo. 13, vol. 2, julio a diciembre de 2018. Páginas 53-68. ISSN 1851-6297 (DESDE DICIEMBRE DE 2006 A DICIEMBRE DE 2017). ISSN 2362-3349 (EN LINNEA). ANÁLISIS DEL SISTEMA DE

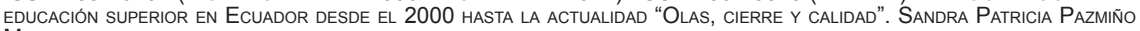
Moscoso.

gurar la calidad de la educación superior; buscó la democratización del acceso a la universidad, pero también de las prácticas gubernativas y administrativas de las instituciones; consolidó la educación superior como un bien público y planteó la necesidad de una mayor regulación estatal para asegurar que esto se cumpla.

Para lograr su cometido, la LOES creó tres instituciones destinadas a planificar y ejecutar las políticas públicas, y regular el Sistema de Educación Superior: la Secretaría Nacional deEducación Superior, Ciencia, Tecnología e Innovación (SENESCYT) como el Ministerio rector de la política pública de educación superior, a cuya creación se resistió el corporativismo universitario; el Consejo de Educación Superior (CES), que autoriza la apertura de carreras y programas, expide normativas y reglamentos para regular el campo, y sanciona las ilegalidades; y, el Consejo de Evaluación, Acreditación, y Aseguramiento de la Calidad de la Educación Superior (CEAACES) que remplaza al CONEA y asume una serie de competencias adicionales que incluyen la evaluación, acreditación y categorización institucional y carreras, además de la certificación de un número significativo de profesiones, mediante exámenes habilitantes, una función que previamente estuvo en manos de los colegios profesionales.

Uno de los artículos más polémicos de la LOES fue la Disposición Transitoria Tercera, que recuperó con fuerza el cumplimiento del Mandato 14, le dio al informe del CONEA un reconocimiento legal y retomó su propuesta inicial de que las 26 universidades categoría "E" sean "depuradas". Para lograr esta depuración, dicha Disposición estableció una nueva evaluación, que debía hacerse en los 18 meses posteriores a la promulgación de la Ley, es decir hasta el 12 abril de 2012. Las 26 universidades y escuelas politécnicas categoría "E" que no superen la evaluación del CEAACES tendrían que cerrarse (5).

\section{La evaluación}

El análisis del "entorno del aprendizaje" del CEAACES evaluó a la universidad en su conjunto, sobre la base de cuatro criterios: (i) academia, (ii) currículo e investigación, (iii) soporte pedagógico y (iv) gestión y política institucional (Long, 2013, p. 23).

También se midió si el ejercicio de la cátedra se daba en el área de la especialización de los docentes (un grave problema en Ecuador), el tiempo de dedicación y el nivel de interacción de los docentes con sus pares y con los estudiantes, entre otros. Estos indicadores buscaban identificar la existencia o ausencia de una comunidad académica, columna vertebral de la universidad, y sancionar la práctica recurrente de depender excesivamente de la contratación de los llamados "profesores taxi", que iban de universidad en universidad facturando por hora de clase dictada, sin tener lazos fuertes con ninguno de los entornos de aprendizaje con los que estaban vinculados, y precarizados en sus condiciones laborales. 
Revista de la Escuela de Ciencias de la Educación, año 14, nRo. 13, vol. 2, julio a diciembre de 2018. PÁginas 53-68. ISSN 1851-6297 (DESDE DICIEMBRE DE 2006 A DICIEMBRE DE 2017). ISSN 2362-3349 (EN LINEA). ANÁLISIS DEL SISTEMA DE EdUCACIÓN superior en Ecuador desde el 2000 hasta la actualidad "Olas, cierre y Calidad". Sandra Patricia Pazmiño Moscoso.

Una vez finalizadas las visitas, el CEAACES dio paso a los exámenes de evaluación de resultados de aprendizaje de los estudiantes de último año. Si se podía comprobar el bajo nivel del aprendizaje significaba, por ejemplo, un riesgo o potencial daño para la sociedad, este sería un motivo lo suficientemente justificado para optar por el cierre de cualquier institución.

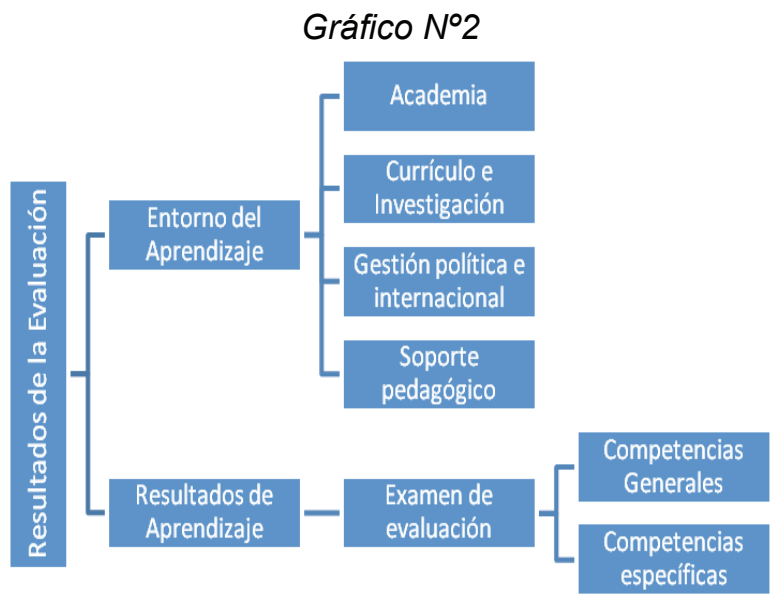

Gráfico N²: Modelo de evaluación

Fuente: CEAACES

El examen, tomado el 4 de marzo de 2012, estuvo dividido en dos partes: un examen de competencias generales y un examen de competencias específicas. Los estudiantes contaron con un total de cuatro horas para la aplicación de ambas pruebas.

Cabe recalcar que este examen marcó un hito en la historia del país. Fue la primera vez que se hizo una prueba parecida a un examen de egreso en 16 carreras de 26 universidades y en 17 de las 24 provincias del país. La aplicación contó con la participación de más de 500 aplicadores presentes en las aulas. La inviolabilidad de los materiales de aplicación y de las hojas de respuesta se realizó con el resguardo de las Fuerzas Armadas y la Policía Nacional.

\section{El cierre}

Tomando en consideración los resultados arrojados por la evaluación del entorno y de los resultados de aprendizaje, el equipo técnico del CEAACES elaboró un Informe Preliminar de Evaluación, que fue entregado a cada una de las 26 universidades. Luego, mediante un proceso de apelaciones y audiencias públicas, se abrió un periodo para la corrección y consolidación de 
Revista de la Escuela de Ciencias de la Educación, año 14, nRo. 13, vol. 2, julio a diciembre de 2018. Páginas 53-68. ISSN 1851-6297 (DESDE DICIEMBRE DE 2006 A DICIEMBRE DE 2017). ISSN 2362-3349 (EN LINNEA). ANÁLISIS DEL SISTEMA DE

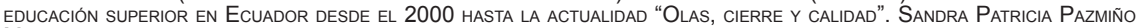
Moscoso.

los datos. Finalmente, el CEAACES elaboró un Informe Final de Evaluación para cada una de las 26 universidades categoría "E".

Los resultados de los exámenes fueron igualmente alarmantes. Tanto los resultados en las diferentes carreras examinadas cuanto los resultados del examen de competencias generales fueron muy insuficientes. Este último dejó al descubierto una población estudiantil con grandes dificultades a la hora de leer un texto y procesar de allí ideas matrices, argumentos, relaciones causales y peor aún fallas imperdonables en el campo de la Medicina.

EI CEAACES categorizó a las 26 universidades en tres grupos: 1) "aceptable", 2) "parcialmente aceptable" y 3) "no aceptable".

En el grupo 1 o "aceptable" se ubicaron tres universidades. En el grupo 20 "parcialmente aceptable" se situaron ocho que pudieron permanecer en el Sistema de Educación Superior pero, al detectarse problemas serios en el transcurso de la evaluación, se recomendó una depuración interna que significó sujetarlas a ciertas interdicciones: no podían matricular a nuevos estudiantes.

En el grupo 3 o "no aceptable" se ubicaron las 14 universidades que fueron suspendidas definitivamente.

La suspensión definitiva de 14 universidades y escuelas politécnicas tuvo varias consecuencias. Algunas autoridades académicas de las universidades cerradas recurrieron como, era de esperar, a la vía judicial. Hasta marzo del 2013, el CEAACES ha enfrentado siete demandas judiciales de diferente índole, aunque de los procesos judiciales en curso, ninguno ha podido prosperar en la reversión de las resoluciones de cierre.

\section{Plan de contingencia}

La mayor dificultad del cierre de las universidades fue sin duda el Plan de Contingencia contemplado en la Disposición Transitoria Tercera de la LOES. La suspensión de las 14 universidades significó que 43.000 estudiantes de estas instituciones, cerca del $7,5 \%$ de la población estudiantil universitaria del país- tenga que acogerse al Plan de Contingencia elaborado por el CES para continuar sus estudios, de acuerdo a uno de tres mecanismos: 1) para los estudiantes de último año de las universidades, la culminación de sus estudios en la institución intervenida por el Estado hasta su cierre definitivo, antes de transcurrir un año; 2) reubicación y continuación de los estudios en una carrera vigente de otra institución de educación superior; y, 3) continuación de estudios en una carrera de titulación especial (6).

Otro dato relevante es el de la continuidad de los estudios, que se mide a través de la tasa de continuidad, definida como la relación entre los estudiantes matriculados y los estudiantes inscritos en el Plan de Contingencia, y que se expresa mediante porcentajes. 


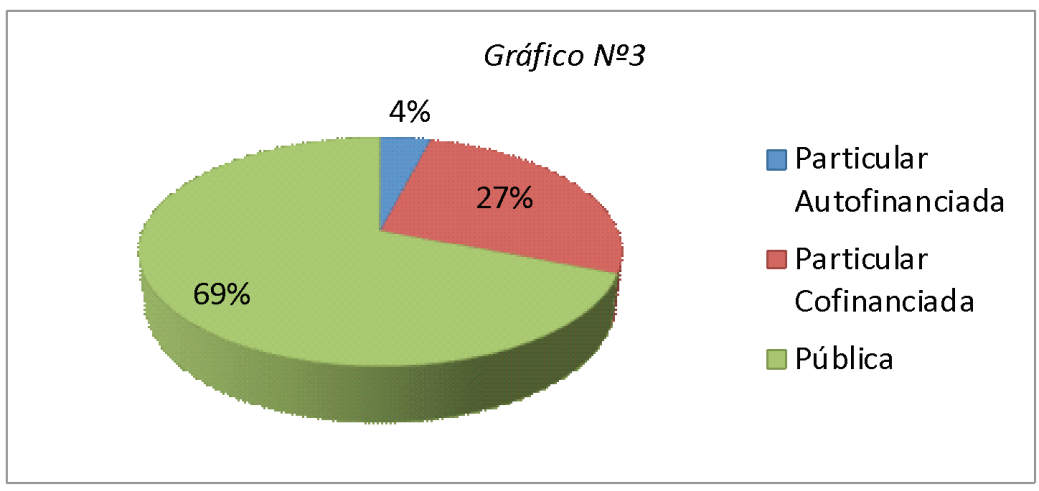

Gráfico N³: Distribución de matrícula por institución y tipo de financiamiento 2012 Fuente: CES

Los estudiantes que se matricularon en las universidades categoría "E" durante la prohibición, sea por ingenuidad o porque pensaban que el CEAACES nunca llegaría a cerrarlas, no pudieron acogerse al Plan de Contingencia.

En ciertos casos, la única oferta similar existía en una universidad de una provincia lejana, lo que causó cierto malestar entre estudiantes poco proclives a trasladarse de provincia. En otros casos, la carrera simplemente no existía en otra universidad del país, por lo que un número importante de estudiantes tuvieron que ser reubicados en carreras afines o genéricas.

Aquellos estudiantes que se reubicaron en universidades públicas fueron, no obstante, muy beneficiados, ya que gozaron del derecho a la gratuidad de la educación superior, como lo manda la Constitución del Ecuador.

\section{El cambio en la Educación Superior}

El Sistema Nacional de Evaluación y Acreditación de la Educación Superior (CEAACES) está integrado por todas las instituciones de educación superior, de las cuales 62 son universidades y escuelas politécnicas, 277 institutos superiores técnicos y tecnológicos, el Consejo Nacional de Evaluación y Acreditación de la Educación Superior (CES), como organismo acreditador; el componente legal que lo integra la Constitución Política de la República, la Ley Orgánica de Educación Superior (LOES), el Reglamento General del CEAACES; y, por el componente técnico que lo integran las características y 18 estándares de calidad, guías de autoevaluación, Manual de Evaluación Externa y otros instructivos y documentos técnicos (7).

La Secretaría Nacional de Educación Superior, Ciencia, Tecnología e Innovación (SENESCYT), dando cumplimiento a lo dispuesto en el artículo 81 
Revista de la Escuela de Ciencias de la Educación, año 14, nRo. 13, vol. 2, julio a diciembre de 2018. Páginas 53-68. ISSN 1851-6297 (DESDE DICIEMBRE DE 2006 A DICIEMBRE DE 2017). ISSN 2362-3349 (EN LíNEA). ANÁLISIS DEL SISTEMA DE

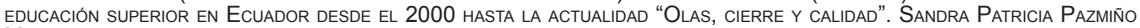
Moscoso.

de la Ley Orgánica de Educación Superior, implementó el Sistema Nacional de Nivelación y Admisión (SNNA), que observa los principios de igualdad de oportunidades, mérito y capacidad (8).

En este marco se creó el Examen Nacional para la Educación Superior (ENES), que explora las habilidades básicas de razonamiento matemático, verbal y lógico. Los resultados orientan al estudiante para que elija la carrera que desea estudiar obteniendo desde un mínimo de 800 puntos; definiéndose valores específicos para cada carrera, por ejemplo medicina 950 a 1000 puntos (9).

En febrero del 2012 se aplicó la primera versión del ENES a los 57.912 bachilleres aspirantes a ingresar a cinco IES del país: Universidad de Guayaquil, Técnica de Babahoyo, Universidad Nacional de Milagro, Escuela Politécnica del Ejército e Instituto Tecnológico Central Técnico.

Sólo aquellos estudiantes interesados en acceder a la educación superior pública deberán rendir dicho examen.

Sin embargo, para el año 2013, no pudieron ingresar más de 11.000 jóvenes ecuatorianos/as, a las universidades hecho importante en la historia de la educación en el Ecuador, no completaron el mínimo de 800 puntos básicos, quedando fuera de este proceso que por primera vez se lo hacía en la nación.

En el año 2012 no pudieron ingresar a nuestras Universidades más de 9.000 jóvenes ecuatorianos/as. En el año 2013 más de 6.000 jóvenes ecuatorianos/as tampoco pudieron ingresar a las Universidades, para el año 2015 no lograron ingresar más de 5.000 jóvenes ecuatorianos/as.

Para el año 2017 con el afán de mejorar la situación respecto al ingreso a las IES públicas, el examen Ser Bachiller (examen que rinden los estudiantes para graduarse) será el instrumento que usará la Senescyt para el ingreso a las universidades en reemplazo al Examen Nacional para la Educación Superior (ENES) ${ }^{\circ}$, esta prueba tendrá un peso del $30 \%$ de la nota de grado y en caso de que el estudiante no obtenga 7 puntos deberá rendir un supletorio. De no aprobar ese examen, el estudiante se podrá presentar al examen de grado (por una única oportunidad) en el siguiente ciclo.

\section{Acreditación}

La evaluación y la acreditación deben ser entendidas como una política pública para garantizar una expansión de la educación superior con calidad académica y relevancia social (Luce y Morosini, 2003).

Finalmente, en la actualidad se encuentran en proceso de acreditación todas las carreras que se ofertan en las Universidades del Ecuador, donde dentro de una misma normativa el CEAACES realiza dos mecanismos de evaluación, el primero sobre Entornos del Aprendizaje consistente en un conjunto de criterios y estándares comunes que aplican, en principio, a todas las ca- 
Revista de la Escuela de Ciencias de la Educación, año 14, nRo. 13, vol. 2, julio a diciembre de 2018. PÁginas 53-68. ISSN 1851-6297 (DESDE DICIEMBRE DE 2006 A DICIEMBRE DE 2017). ISSN 2362-3349 (EN LINEA). ANÁLISIS DEL SISTEMA DE

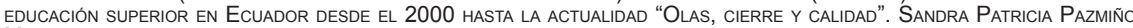
Moscoso.

rreras de las universidades y escuelas politécnicas del país; estos constituyen el modelo de evaluación genérico de carreras. Finalmente, considerando las particularidades de cada área del conocimiento, se formulan modelos de evaluación específicos para cada una de las carreras.

Y el segundo que consiste en el Examen Nacional de Evaluación de Carreras evalúa los resultados de aprendizaje de las carreras que están en proceso de evaluación. Se aplica a los estudiantes de último año y es uno de los dos mecanismos que determinan la acreditación de una carrera (conjuntamente con la evaluación de entorno de aprendizaje).

El proceso de evaluación de entorno de aprendizaje de una carrera consta de las siguientes etapas esenciales: autoevaluación, carga de información, evaluación documental, visita in situ, rectificaciones, apelaciones e informe final.

Los modelos de evaluación son construidos con la participación de las universidades y escuelas politécnicas. Las discusiones son sobre la base de una propuesta inicial realizada por expertos en las áreas del conocimiento a evaluarse.

Con la publicación de la Ley Orgánica de Educación Superior (LOES) en el 2010, el Ecuador inició un proceso orientado a garantizar el derecho a la Educación Superior de calidad, que propenda a la excelencia, al acceso universal, a la permanencia y egreso sin discriminación.

Durante los últimos años, el acceso a la Educación Superior se ha incrementado en varios puntos porcentuales, lo que muestra un enorme esfuerzo por ampliar la cobertura. El origen de este cambio radica en acciones progresivas que permiten ir eliminando las barreras de acceso.

Los sectores más pobres del país han duplicado su acceso a la Educación Superior. Los Sistemas de becas, créditos educativos, estímulos son factores que han permitido el acceso a hijos de familias pobres, generando de alguna manera la ruptura de la pobreza y un resultado social importante, sin embargo, de la misma manera cientos de jóvenes no han podido aprobar el ENES en años anteriores y hasta la actualidad se encuentran sin acceso a la Educación Superior en instituciones Públicas.

Recientemente, Ecuador cuenta con Universidades y Escuelas Politécnicas con excelencia académica, reconocidas a nivel nacional e internacional. Sin embargo, también existe un número significativo de instituciones de Educación Superior, que se encuentran en proceso de acreditación para alcanzar los estándares establecidos.

\section{Conclusión}

Se ha definido la situación actual del Ecuador en el sistema educacional superior, siendo realistas, detallando el proceso de cierre de 14 universidades y escuelas politécnicas realizado por el CEAACES en cumplimiento de la disposición transitoria tercera de la LOES. 
Revista de la Escuela de Ciencias de la Educación, año 14, nRo. 13, vol. 2, julio a diciembre de 2018. Páginas 53-68. ISSN 1851-6297 (DESDE DICIEMBRE DE 2006 A DICIEMBRE DE 2017). ISSN 2362-3349 (EN LINNEA). ANÁLISIS DEL SISTEMA DE

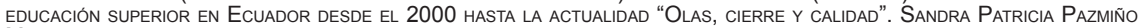
Moscoso.

Resaltando que la citada tercera ola de creación de universidades privadas se denominaría en particular como una estafa académica con fines de lucro, que aconteció en respuesta a fenómenos recurrentes políticos de muchos países de la región y del mundo, en el contexto de una Latinoamérica atravesada por problemas de carencia de calidad y pertinencia en la que la experiencia de Ecuador es particularmente digna de análisis.

Finalmente, después del cierre de las 14 universidades, al implementarse la evaluación por parte de entes reguladores del Sistema de Educación Superior, todas las instituciones vienen realizando renovados esfuerzos en distinto grado y con diversos resultados.

Siendo importante que las distintas actividades que se desarrollan en las Instituciones Públicas rectoras de la educación superior; sean ejecutadas de forma coordinada con las IES para poder afrontar conjuntamente los distintos desafíos con miras a un futuro educativo de desarrollo y calidad.

\section{Declaración de conflicto de interés}

Ninguna

\section{Financiamiento}

Propio de la autora

\section{Referencias}

(1) Congreso Ecuador, (2000), Ley de Educación Superior-Ley No. 16, RO/77, Quito - Ecuador. p.7

(2) Asamblea Nacional Constituyente, (2014), Mandato Constituyente № 14, Ecuador:. CEAACES. Recuperado de http://www.ceaaces.gob.ec/sitio/wp-content/ uploads/2014/02/INFORME_FINAL_UNIVERSIDADES_M141.pdf

(3) CONEA, (2009),Base de datos, Proyecto Mandato 14. "Evaluación de desempeño institucional de las Universidades y Escuelas Politécnicas del Ecuador", Quito Ecuador

(4) Asamblea Nacional Constituyente, (2010) Ley Orgánica de Educación Superior LOES, Quito - Ecuador, Artículo 80.p. 10-11.

(5) Asamblea Nacional Constituyente, (2010) Ley Orgánica de Educación Superior LOES, p. 36.

(6) CES, (2013), Reglamento del Plan de Contingencia para las y los estudiantes de las Universidades y Escuelas Politécnicas de Categoría E que se suspendan definitivamente (Codificación), Quito - Ecuador. Artículo 13 p. 6

(7) CEAACES, (2014). Reglamento de Evaluación, Acreditación y Categorización de Carreras de las Instituciones de Educación Superior, Quito - Ecuador. Artículo 5 p. 3.

(8) SENESCYT, (2015). Sistema Nacional de Nivelación y Admisión Asignación de cu- 
pos, un proceso transparente y meritocrático. Quito.: SNNA. Recuperado de:http:// www.snna.gob.ec/wp-content/themes/institucion/comunicamos_noticias93.php

(9) SENESCYT. (2017). Reformas al Reglamento del Sistema Nacional de Nivelación y Admisión SNNA Artículo 1, p. 2. Quito.: Recuperado de:http://www.snna.gob.ec/ dwpages/Descargas/Acuerdo_SENESCYT_2017_124_Reformas_Reglamento_ SNNA.pdf

\section{Referencias de tablas y gráficos}

- Tabla 1: CONEA (2008). Sistema de Educación Superior del Ecuador 2008.

- Fuente: Acosta N (2016), "El efecto de la educación gratuita universitaria sobre la asistencia a clases y en el mercado laboral: evidencia para el Ecuador", Quito - Ecuador

- Gráfico 1: INEC (2008), Incremento de matrícula de estudiantes en universidades privadas (1998 - 2008).

- Fuente: Acosta N (2016), "El efecto de la educación gratuita universitaria sobre la asistencia a clases y en el mercado laboral: evidencia para el Ecuador", Quito - Ecuador

- Gráfico N²: CEAACES (2012), Modelo de evaluación.

- Fuente: CEAACES, Reglamento de Evaluación, Acreditación y Categorización de Carreras de las Instituciones de Educación Superior, Quito - Ecuador.

- Gráfico N³: CES(2013), Distribución de matrícula porinstitución y tipo de financiamiento 2012. Fuente: Consejo de Educación Superior, Reglamento para garantizar el derecho a la continuidad de los estudios regulares de las y los estudiantes de las Universidades y Escuelas Politécnicas suspendidas definitivamente por el CEAACES. Tomado de: http://www.ces.gob.ec/doc/Reglamentos/rpc-so-45-no\%20467-2013.pdf 